\title{
Anti-Ramsey problems for $t$ edge-disjoint rainbow spanning subgraphs: cycles, matchings, or trees
}

\author{
Sogol Jahanbekam*and Douglas B. West ${ }^{\dagger}$
}

Revised April 2015

\begin{abstract}
We seek the maximum number of colors in an edge-coloring of the complete graph $K_{n}$ not having $t$ edge-disjoint rainbow spanning subgraphs of specified types. Let $c(n, t), m(n, t)$, and $r(n, t)$ denote the answers when the spanning subgraphs are cycles, matchings, or trees, respectively. We prove $c(n, t)=\left(\begin{array}{c}n-1 \\ 2\end{array}\right)+t$ for $n \geq 8 t-1$ and $m(n, t)=\left(\begin{array}{c}n-2 \\ 2\end{array}\right)+t$ for $n>4 t+10$. We prove $r(n, t)=\left(\begin{array}{c}n-2 \\ 2\end{array}\right)+t$ for $n>2 t+\sqrt{6 t-\frac{23}{4}}+\frac{5}{2}$ and $r(n, t)=\left(\begin{array}{l}n \\ 2\end{array}\right)-t$ for $n=2 t$. We also provide constructions for the more general problem in which colorings are restricted so that colors do not appear on more than $q$ edges at a vertex.

Keywords: spanning subgraph, rainbow, anti-Ramsey number, perfect matching, Hamiltonian cycle, spanning tree MSC code: 05C55, 05C35, 05C38, 05C04
\end{abstract}

\section{Introduction}

The general anti-Ramsey problem asks for the maximum number of colors in an edge-coloring of the complete graph $K_{n}$ not containing a rainbow subgraph in a specified family $\mathcal{G}$; the notation for the answer is $\operatorname{AR}(n, \mathcal{G})$. Early results where $\mathcal{G}$ consists of one graph were surveyed in [10]. Notable among these is the result of Montellano-Ballesteros and NeumannLara [20]; they proved a long-standing conjecture of Erdős, Simonovits, and Sós [9] by computing $\operatorname{AR}\left(n, C_{k}\right)$. Among larger (but fixed) families, Jiang and West [17] determined the anti-Ramsey number of the family of all trees with $m$ edges.

*jahanbe1@illinois.edu; University of Illinois, Urbana, IL. Research supported in part by National Science Foundation grant DMS 09-01276.

†west@math.uiuc.edu; Zhejiang Normal University, Jinhua, China, and University of Illinois, Urbana, IL. Research supported by Recruitment Program of Foreign Experts, 1000 Talent Plan, State Administration of Foreign Experts Affairs, China. 
More recent work seeks rainbow subgraphs that grow with $n$, such as spanning rainbow subgraphs of special types. Bialostocki and Voxman [4] showed that the maximum number of colors in an edge-coloring of $K_{n}$ with no rainbow spanning tree is $\left(\begin{array}{c}n-2 \\ 2\end{array}\right)+1$. Haas and Young [13] showed that the maximum number with no rainbow spanning matching (for even $n$ ) is $\left(\begin{array}{c}n-3 \\ 2\end{array}\right)+2$ when $n \geq 14$ (they also determined the answer for smaller $n$ ). Their results match the construction given (and conjectured optimal) in [11] (a longer proof of the solution appeared earlier in [7]).

We extend such problems by seeking the maximum number of colors in an edge-coloring of $K_{n}$ not having $t$ edge-disjoint rainbow spanning subgraphs of particular types. Let these values be $m(n, t)$ for spanning (perfect) matchings, $c(n, t)$ for spanning (Hamiltonian) cycles, and $r(n, t)$ for spanning trees. Our result for matchings is the following.

Theorem 1.1. If $n$ is even and $n>t \geq 2$, then $m(n, t) \geq\left(\begin{array}{c}n-2 \\ 2\end{array}\right)+t$. Equality holds when $n>4 t+10$.

We believe that equality holds whenever $n>t \geq 2$ if $n>4$; note that $m(n, t)=\left(\begin{array}{c}n-2 \\ 2\end{array}\right)+t+1$ when $n=4$. The formula does not reduce to the result of Haas and Young [13] when $t=1$. Nevertheless, after exceeding the maximum number of colors that can avoid two edge-disjoint rainbow perfect matchings, each additional color forces one more rainbow perfect matching. This behavior is the same for cycles and trees, but starting with $t=1$.

Because we are discussing spanning subgraphs of various types, we maintain consistency by writing "spanning cycle" rather than "Hamiltonian cycle". Trivially, $c(n, t) \geq m(n, 2 t)$ when $n$ is even. Our result about cycles is the following.

Theorem 1.2. If $t \geq 1$ and $n \geq 2 t+2$, then $c(n, t) \geq\left(\begin{array}{c}n-1 \\ 2\end{array}\right)+t$. Equality holds when $n \geq 8 t-1$.

Here the formula remains valid when $t=1$, and we conjecture that it is the correct value whenever $n \geq 2 t+2$. Because the proof of this is easier and uses more familiar ideas, we study the spanning cycles before the matchings.

For $t$ edge-disjoint rainbow spanning trees, our formula reduces to the result of Bialostocki and Voxman [4] in the special case $t=1$. Akbari and Alipour [1] proved $r(n, 2)=\left(\begin{array}{c}n-2 \\ 2\end{array}\right)+2$ for $n \geq 6$. If $n<2 t$, then $K_{n}$ does not have enough edges for $t$ edge-disjoint spanning trees. For each $t$, we determine $r(n, t)$ except for roughly $\sqrt{6 t}$ values of $n$. We conjecture that equality in the theorem below holds in fact for $n \geq 2 t+2 \geq 6$ (when $n=2 t+1$, there is a construction with $\left(\begin{array}{c}n-2 \\ 2\end{array}\right)+2 t-1$ colors that avoids $t$ edge-disjoint rainbow spanning trees).

Theorem 1.3. If $n>2 t \geq 2$, then $r(n, t) \geq\left(\begin{array}{c}n-2 \\ 2\end{array}\right)+t$. Equality holds when $n>2 t+\sqrt{6 t-\frac{23}{4}}+\frac{5}{2}$. If $n=2 t$, then $r(n, t)=\left(\begin{array}{l}n \\ 2\end{array}\right)-t$.

Although we have determined $c(n, t), m(n, t)$, and $r(n, t)$ exactly except for small $n$, it is a bit unsatisfying that the answers are so close to $\left(\begin{array}{l}n \\ 2\end{array}\right)$ and that in extremal constructions avoiding $t$ rainbow subgraphs almost all the edges at one or two vertices have the same color. 
More general problems exclude such colorings. LeSaulnier and West [19] used the term $q$-tolerant to describe edge-colorings where no vertex has more than $q$ incident edges with the same color. Let $c_{q}(n, t), m_{q}(n, t)$, and $r_{q}(n, t)$ denote the maximum number of colors in a $q$-tolerant edge-coloring of $K_{n}$ not having $t$ edge-disjoint rainbow spanning cycles, matchings, or trees, respectively.

Proper edge-colorings are just 1-tolerant edge-colorings. Brualdi and Hollingsworth [5] conjectured that every proper $(n-1)$-edge-coloring of $K_{n}$ decomposes into rainbow spanning trees (for $n \geq 6$ ). They proved that two edge-disjoint rainbow spanning trees can always be found. Horn [15] proved that $\epsilon n$ edge-disjoint rainbow spanning trees can be found, for some positive constant $\epsilon$.

Kaneko, Kano, and Suzuki [18] conjectured more generally that every properly edgecolored complete graph (with any number of colors) decomposes into rainbow spanning trees, and they proved that three such trees can always be found. Akbari and Alipouri [1] showed that two edge-disjoint rainbow spanning trees can be found even when the coloring is not proper, as long as no color appears more than $n / 2$ times (this condition is stronger than $n / 2$-tolerance). Carraher, Hartke, and Horn [6] proved that there are at least $\lfloor n /(323 \log n)\rfloor$ such trees when $n \geq 65000$.

For rainbow cycles, Akbari et al. [2] guaranteed that a proper edge-coloring of $K_{n}$ contains a rainbow cycle of length at least $n / 2-1$. Gyárfás et al. [12] improved this to $(4 / 7-o(1)) n$.

In general, these problems seem quite difficult when $q=1$. The value for which we solve some of the questions is $q=n-1$, which drops all restrictions on tolerance. In Section 2, we provide constructions for general $q$ to place our results in a broader context. We then prove the upper bounds for Theorems 1.1-1.3 in Sections 3-5.

Most upper bounds for anti-Ramsey problems use a common technique. A representing subgraph in an edge-coloring of $K_{n}$ is a spanning subgraph containing exactly one edge of each color. Our proofs that the constructions are optimal generally proceed by choosing a representing subgraph that is extremal in some sense and proving that it or a graph closely related to it contains the desired subgraph(s).

To obtain the desired subgraphs, we apply various classical tools. For spanning cycles, we use corollaries of the sufficient condition by Chvátal [8] on vertex degrees for spanning cycles. For spanning matchings, we use a recent lower bound [16] for maximum matchings in bipartite graphs. For spanning trees, we use the condition of Nash-Williams [21] for edgedisjoint spanning trees and a classical lower bound on the matching number of a bipartite graph in terms of the minimum degree. 


\section{Construction of $q$-Tolerant Edge-Colorings}

Recall that $c_{q}(n, t), m_{q}(n, t)$, and $r_{q}(n, t)$ are the maximum numbers of colors in a $q$-tolerant edge-coloring of $K_{n}$ not having $t$ edge-disjoint rainbow spanning cycles, matchings, or trees, respectively. The constructions for the three problems are similar.

The join $H \oplus H^{\prime}$ of two graphs $H$ and $H^{\prime}$ is the graph obtained from the disjoint union $H+H^{\prime}$ by making all of $V(H)$ adjacent to all of $V\left(H^{\prime}\right)$. Let $\bar{G}$ denote the complement of a graph $G$, and let $G[S]$ denote the subgraph induced by a vertex subset $S$.

Lemma 2.1. Fix $p, q \in \mathbb{N}$. For positive integers $s$ and $s^{\prime}$ with $s, s^{\prime} \leq p q$, the complete bipartite graph $K_{s, s^{\prime}}$ has a q-tolerant edge-coloring using $p$ colors. If $p q \geq n>p$, then $K_{p} \oplus \bar{K}_{n-p}$ also has such an edge-coloring, as does $K_{p+1} \oplus \bar{K}_{n-p-1}$ if also $p$ is odd, $p q \geq n-1$, and $q \geq 3$.

Proof. The graph $K_{s, s^{\prime}}$ is bipartite with maximum degree at most $p q$; hence it has a proper edge-coloring with at most $p q$ colors. Combining the colors into $p$ sets of size at most $q$ yields a $q$-tolerant edge-coloring with $p$ colors.

If $p q \geq n$ with $p<n$, then $q \geq 2$, and the previous paragraph yields a ( $q-1)$-tolerant edgecoloring with $p$ colors for the subgraph $K_{p, n-p}$ of $K_{p} \oplus \bar{K}_{n-p}$. It remains to add a coloring of the complete subgraph of order $p$. By Vizing's Theorem, this subgraph has a proper coloring using $p$ colors. This adds at most 1 to the multiplicity of each color at each vertex, completing the desired $q$-tolerant edge-coloring. If $p$ is odd and $q \geq 3$, then the same argument works starting with $K_{p+1, n-p-1}$.

In each construction below, we partition the vertex set of $K_{n}$ into sets $S$ and $S^{\prime}$ of sizes $s$ and $s^{\prime}$. We define a $q$-tolerant edge-coloring on the corresponding subgraph $K_{s, s^{\prime}}$ or $K_{s} \oplus \bar{K} s^{\prime}$ using Lemma 2.1 and extend this to a $q$-tolerant edge-coloring of $K_{n}$. In each case, there will be $t-1$ "key" colors used on single edges such that every rainbow spanning subgraph of the desired type must use at least one of these edges. Consequently, the construction will not have $t$ such subgraphs.

When we add new colors on single edges or switch single edges to new colors, the coloring remains $q$-tolerant. Also, we can switch $t-1$ edges to new colors without eliminating existing colors when the switched edges come from color classes whose average size exceeds $t$.

Proposition 2.2. If $n, t, q$ are positive integers such that $n>2 t$ and $q \geq 2$, then $c_{q}(n, t) \geq$ $\left(\begin{array}{c}n-p \\ 2\end{array}\right)+p+t-1$, where $p=\left\lceil\frac{n}{q}\right\rceil$.

Proof. Choose a vertex partition $\left(S, S^{\prime}\right)$ with $|S|=p$ and $\left|S^{\prime}\right|=n-p$. Since $p q \geq n$, Lemma 2.1 yields a $q$-tolerant edge-coloring of $K_{p} \oplus \bar{K}_{n-p}$ using $p$ colors, where $S$ is the $p$-clique. Change the colors on $t-1$ edges incident to $S$ to new "key" colors without eliminating colors. Color the edges of $K_{n}$ induced by $S^{\prime}$ using $\left(\begin{array}{c}n-p \\ 2\end{array}\right)$ additional colors on one edge each. The coloring is $q$-tolerant. Each rainbow spanning cycle uses an edge with a key color, since a cycle avoiding them has at least $p+1$ edges incident to $S$, all confined to a set of $p$ colors. 
Setting $q=n$ yields $p=1$, and then the formula shows that our upper bound for $n \geq 8 t-1$ in Theorem 3.4 is sharp when there are no tolerance restrictions. This holds also for matchings, but the situation is a bit more complicated for matchings because we need two constructions. The necessary condition for the stronger construction suggests the difference between the cases $t=1$ and $t>1$ in the matching problem with unrestricted tolerance, since $\left(\begin{array}{c}1+1 \\ 2\end{array}\right)<t$ if and only if $t>1$.

Proposition 2.3. Let $n, t, q$ be positive integers such that $n$ is even, $n>t$, and $q \geq 2$. If $\left(\begin{array}{c}p+1 \\ 2\end{array}\right)<t$, where $p=\left\lceil\frac{n-1}{q+1}\right\rceil$, then $m_{q}(n, t) \geq\left(\begin{array}{c}n-p-1 \\ 2\end{array}\right)+p+t-1$. When $\left(\begin{array}{c}p+1 \\ 2\end{array}\right) \geq t$ and $q>\sqrt{n}$, there is a weaker bound: $m_{q}(n, t) \geq\left(\begin{array}{c}n-p^{\prime}-1 \\ 2\end{array}\right)+p^{\prime}+t-2$, where $p^{\prime}=\left\lceil\frac{n-3}{q+1}\right\rceil+2$.

Proof. Choose a vertex partition $\left(S, S^{\prime}\right)$ with $|S|=p+1$ and $\left|S^{\prime}\right|=n-p-1$. Since $p q \geq n-p-1$ and $p q \geq p+1$, by Lemma 2.1 there is a $q$-tolerant edge-coloring of $K_{p+1, n-p-1}$ using $p$ colors, where $S$ is the part of size $p+1$. Use new key colors on $t-1$ edges incident to $S$, including all edges induced by $S$. Color the edges of $K_{n}$ induced by $S^{\prime}$ using $\left(\begin{array}{c}n-p-1 \\ 2\end{array}\right)$ additional colors that each appear on one edge. A rainbow spanning matching that avoids the key colors matches all $p+1$ vertices of $S$ into $S^{\prime}$, but such edges have at most $p$ colors. Hence each rainbow spanning matching must use a key color.

When $\left(\begin{array}{c}p+1 \\ 2\end{array}\right) \geq t$, we cannot color all edges induced by $S$ with key colors. Instead, choose a vertex partition $\left(S, S^{\prime}\right)$ with $|S|=p^{\prime}+1$ and $\left|S^{\prime}\right|=n-p^{\prime}-1$; note that $p^{\prime}>p$. The definition of $p^{\prime}$ yields $\left(p^{\prime}-2\right) q \geq n-p^{\prime}-1$. By Lemma 2.1 , there is a $q$-tolerant edge-coloring of $K_{p^{\prime}+1, n-p^{\prime}-1}$ using $p^{\prime}-2$ colors, where $S$ is the part of size $p^{\prime}+1$. Color all the edges induced by $S$ with one additional color, but change the color on $t-1$ of these edges to new key colors. The coloring is still $q$-tolerant, since $q>\sqrt{n}$ yields $q \geq p^{\prime}$. Color the edges of $K_{n}$ induced by $S^{\prime}$ using $\left(\begin{array}{c}n-p-1 \\ 2\end{array}\right)$ additional colors that each appear on one edge. A rainbow spanning matching that avoids the key colors can only use one edge within $S$, since those edges all have the same color. Hence it uses at least $p^{\prime}-1$ edges joining $S$ to $S^{\prime}$, but only $p^{\prime}-2$ colors appear on those edges. Hence each rainbow spanning matching must use a key color.

Proposition 2.4. If $n, t, q$ are positive integers such that $n>t$ and $q \geq 3$, then $r_{q}(n, t) \geq$ $\left(\begin{array}{c}n-p-1 \\ 2\end{array}\right)+p+t-1$ if $p$ is odd, where $p$ is the smallest odd integer that is at least $\left\lceil\frac{n-1}{q}\right\rceil$.

Proof. Choose a vertex partition $\left(S, S^{\prime}\right)$ with $|S|=p+1$ and $\left|S^{\prime}\right|=n-p-1$. Since $p q \geq n-1$ and $p$ is odd, by Lemma 2.1 there is a $q$-tolerant edge-coloring of $K_{p+1} \oplus \bar{K}_{n-p-1}$ using $p$ colors, where $S$ is the clique of order $p+1$. Change the colors on $t-1$ edges incident to $S$ to new key colors without eliminating any colors. Color the edges of $K_{n}$ induced by $S^{\prime}$ using $\left(\begin{array}{c}n-p-1 \\ 2\end{array}\right)$ additional colors that each appear on one edge. Every spanning tree has at least $p+1$ edges incident to $S$. If it uses no key colors, then these edges are restricted to $p$ colors. Hence every rainbow spanning tree uses a key color. 


\section{Rainbow Spanning Cycles}

When $n \leq 2 t$, a rainbow edge-coloring of $K_{n}$ does not have $t$ edge-disjoint spanning cycles, so then $c(n, t)=\left(\begin{array}{l}n \\ 2\end{array}\right)$. For $n>2 t$, the question becomes nontrivial. The lower bound construction is valid whenever $n>2 t$, but our proof of optimality requires $n \geq 8 t-1$. We conjecture that the construction is optimal for $n>2 t$. Our proof of the lower bound uses a well-known result of Chvátal [8] giving a sufficient condition for a spanning cycle.

Theorem 3.1 (Chvátal [8]). Let $G$ be a graph with vertex degrees $d_{1}, \ldots, d_{n}$ in nondecreasing order. If $d_{i}>i$ or $d_{n-i} \geq n-i$ whenever $i<n / 2$, then $G$ is Hamiltonian.

We need a less familiar corollary, stated in [3, p. 218], for which we review the proof. A graph $G$ is Hamiltonian-connected if for all $u, v \in V(G)$, there is a spanning $u, v$-path.

Corollary 3.2. Let $G$ be a graph with vertex degrees $d_{1}, \ldots, d_{n}$ in nondecreasing order. If $d_{i}>i+1$ or $d_{n-i} \geq n-i+1$ whenever $i<(n-1) / 2$, then $G$ is Hamiltonian-connected.

Proof. To obtain a spanning $u, v$-path in $G$, form $G^{\prime}$ from $G$ by adding a new vertex $w$ with neighborhood $\{u, v\}$. The condition $d_{1}>2$ or $d_{n-1} \geq n$ yields $d_{1} \geq 3$, so $d_{G^{\prime}}(w)=\delta\left(G^{\prime}\right)$. Letting $n^{\prime}=n+1$ and letting $d_{1}^{\prime}, \ldots, d_{n^{\prime}}^{\prime}$ be the vertex degrees in $G^{\prime}$ in nondecreasing order, we have $d_{1}^{\prime}=2$ and $d_{i}^{\prime} \geq d_{i-1}$. Thus $d_{i}^{\prime}>i$ or $d_{n^{\prime}-i} \geq n^{\prime}-i$ whenever $i<n^{\prime} / 2$. By Chvátal's Theorem, $G^{\prime}$ has a spanning cycle $C$, and $C-w$ is a spanning $u, v$-path in $G$.

Corollary 3.2 yields a tool that we will use in proving our upper bounds for both the cycle problem and the matching problem.

Lemma 3.3. Let $G$ be a graph with vertex degrees $d_{1}, \ldots, d_{n}$ in nondecreasing order. If $d_{i} \geq i+2 j$ or $d_{n-i} \geq n-i+2 j-1$ whenever $i<(n-1) / 2$, then $G$ has $j$ edge-disjoint spanning paths with any $j$ specified vertex pairs as endpoints.

Proof. Setting $j=1$ yields the statement of Corollary 3.2. For larger $j$, Corollary 3.2 still yields one such path. Deleting its edges reduces each vertex degree by at most 2 . Hence the claim follows by induction on $j$.

Recall that in an edge-coloring $\phi$ of $K_{n}$, a representing subgraph is a spanning subgraph of $K_{n}$ whose edge set consists of one edge of each color used in $\phi$.

Theorem 3.4. If $t \geq 2$ and $n \geq 8 t-1$, then $c(n, t)=\left(\begin{array}{c}n-1 \\ 2\end{array}\right)+t$.

Proof. By Proposition 2.2 with $p=1$, it remains only to prove the upper bound. Given an

edge-coloring of $K_{n}$ using $\left(\begin{array}{c}n-1 \\ 2\end{array}\right)+t+1$ different colors, we will find $t$ edge-disjoint rainbow spanning cycles. Choose $G$ to be one of the representing subgraphs having the largest minimum degree. Let $d_{1}, \ldots, d_{n}$ be the vertex degrees in $G$, in nondecreasing order. To facilitate application of Theorem 3.1 and Lemma 3.3, we want $d_{i} \geq i+2 t$ for $i \leq(n-1) / 2$. 
If $d_{i}<i+2 t$, then at most $i(2 t+i-1)$ edges of $G$ are incident to the $i$ lowest-degree vertices in $G$. At most $\left(\begin{array}{c}n-i \\ 2\end{array}\right)$ edges are not incident to them, so together $\left(\begin{array}{c}n-1 \\ 2\end{array}\right)+t+1 \leq i(2 t+i-1)+\left(\begin{array}{c}n-i \\ 2\end{array}\right)$. The inequality simplifies to $(i-1)\left(n-2 t-\frac{3}{2} i-1\right) \leq t-1$. Since the left side is quadratic in $i$ with negative curvature, the inequality fails throughout an interval for $i$ if and only if it fails at the ends. It always holds when $i=1$. For $i=2$, it holds if and only if $n \leq 3 t+3$. For $i=(n-1) / 2$ and $n \geq 2 t+1$, it holds if and only if $n-2 t-1 \leq \frac{3}{2} i$, which simplifies to $n \leq 8 t-2$. We conclude that $d_{i} \geq i+2 t$ for $2 \leq i \leq \frac{n-1}{2}$ when $n \geq 8 t-1$.

Now consider $d_{1}$. If $d_{1} \geq 2 t$, then iteratively applying Theorem 3.1 yields $t$ spanning cycles in $G$, as desired. Hence we may assume $d_{1}<2 t$. Since $|E(G)|=\left(\begin{array}{c}n-1 \\ 2\end{array}\right)+t+1$, we have $d_{1} \geq t+1$. The inequalities $d_{i} \geq i+2 t$ still hold for $2 \leq i \leq \frac{n-1}{2}$.

Let $u$ be the vertex with degree $d_{1}$ in $G$. Since $G$ has largest minimum degree among all representing subgraphs, all edges of $K_{n}$ incident to $u$ have colors that appear on an edge incident to $u$ in $G$; otherwise, replacing an edge of $G$ with an edge of the same color at $u$ would increase the minimum degree.

Since $n \geq 3 t$ and $d_{1}<2 t$, we can select edges $e_{1}, \ldots, e_{t}$ incident to $u$ that are not in $G$. For $1 \leq i \leq t$, choose from among $t+1$ edges of different colors incident to $u$ in $G$ an edge $e_{i}^{\prime}$ with color different from $e_{i}$. The pairs $\left\{e_{i}, e_{i}^{\prime}\right\}$ for $1 \leq i \leq t$ form three-vertex rainbow paths through $u$ with endpoints in $G-u$, using colors not in $G-u$. Hence it suffices to find $t$ edge-disjoint spanning paths in $G-u$ having these specifed endpoints.

Let $G^{\prime}=G-u$, let $n^{\prime}=n-1$, and let $d_{1}^{\prime}, \ldots, d_{n^{\prime}}^{\prime}$ be the (nondecreasing) vertex degrees of $G^{\prime}$. Since $d_{G}(u)=d_{1}$, for $i \geq 1$ we have $d_{i}^{\prime} \geq d_{i+1}-1 \geq i+2 t$. By Lemma 3.3, $G^{\prime}$ has $t$ spanning paths that form edge-disjoint rainbow spanning cycles in $G$ with the chosen pairs at $u$.

\section{Rainbow Perfect Matchings}

When $n \leq t$, the rainbow coloring of $E\left(K_{n}\right)$ does not have $t$ edge-disjoint perfect matchings, so then $m(n, t)=\left(\begin{array}{l}n \\ 2\end{array}\right)$. For $n>t$ the question becomes nontrivial. The construction for the lower bound is valid whenever $n>t$, but our proof of optimality requires $n>4 t+10$. We conjecture that the construction is optimal for $n>t$.

In proving the upper bound, we use the lemmas of Section 3 and another result guaranteeing large matchings in bipartite graphs. An $X, Y$-bigraph is a bipartite graph with parts $X$ and $Y$. The matching number $\alpha^{\prime}(G)$ is the maximum size of a matching in $G$.

Theorem 4.1 (Jahanbekam-West [16]). Let $G$ be a graph with vertex degrees $d_{1}, \ldots, d_{n}$ in nondecreasing order. If $G$ is an $X, Y$-bigraph, then $\alpha^{\prime}(G) \geq \max _{k} \min \left\{2 d_{k+2},|X|-k,|Y|-k\right\}$.

We need another corollary of Theorem 3.1 to obtain perfect matchings.

Lemma 4.2. Let $G$ be a graph with vertex degrees $d_{1}, \ldots, d_{n}$ in nondecreasing order, where $n$ is even. If $d_{i} \geq i$ or $d_{n-i} \geq n-i-1$ for $i<n / 2$, then $G$ has a perfect matching. 
Proof. Form $G^{\prime}$ from $G$ by adding a vertex $u$ adjacent to all of $V(G)$. In $G^{\prime}, u$ has highest degree, and the degrees of other vertices increase by 1 from $G$. Letting $d_{1}^{\prime}, \ldots, d_{n^{\prime}}^{\prime}$ be the vertex degrees in $G^{\prime}$, we have $d_{i}^{\prime}>i$ or $d_{n^{\prime}-i}^{\prime}=1+d_{n+1-i} \geq 1+n-i=n^{\prime}-i$. By Theorem 3.1, $G^{\prime}$ has a spanning cycle. Alternate edges along it form a perfect matching in $G$.

In an $n$-vertex graph $G$ with $d_{i} \geq i+t-1$ for $i<n / 2$, Lemma 4.2 guarantees $t$ edge-disjoint perfect matchings. Say that an edge incident to a vertex $w$ is an edge "at" $w$.

Theorem 4.3. If $n$ is even and $n>4 t+10$ with $t \geq 2$, then $m(n, t)=\left(\begin{array}{c}n-2 \\ 2\end{array}\right)+t$.

Proof. By Proposition 2.3 with $p=1$, it suffices to prove the upper bound. It suffices to consider an edge-coloring of $K_{n}$ using exactly $\left(\begin{array}{c}n-2 \\ 2\end{array}\right)+t+1$ colors. We prove that $t$ edgedisjoint rainbow perfect matchings can be found. Among all representing subgraphs having the largest minimum degree, choose $G$ to be one having also the largest value of $d_{2}$ when the vertex degrees are $d_{1}, \ldots, d_{n}$ in nondecreasing order.

By Lemma 4.2, having $d_{i} \geq i+t-1$ for $i \leq n / 2-1$ will yield $t$ edge-disjoint perfect matchings in $G$. Due to a later case, we prefer to require the stronger inequality $d_{i} \geq i+t+1$. As in Theorem 3.4, if $d_{i} \leq i+t$, then at most $i(t+i)$ edges of $G$ are incident to $i$ lowest-degree vertices in $G$. Since $G$ has at most $\left(\begin{array}{c}n-i \\ 2\end{array}\right)$ other edges, $\left(\begin{array}{c}n-2 \\ 2\end{array}\right)+t+1 \leq i(t+i)+\left(\begin{array}{c}n-i \\ 2\end{array}\right)$. The inequality simplifies to

$$
(i-2)\left(n-t-\frac{3 i+7}{2}\right) \leq t+3 .
$$

Since $(*)$ holds when $i \leq 2$, we must consider $d_{1}$ and $d_{2}$ separately later. To prove $d_{i} \geq i+t+1$ for $3 \leq i \leq n / 2-1$, we show that $(*)$ fails on that interval. For fixed $n$ and $t$, the left side is quadratic in $i$ with negative curvature. Hence it exceeds $t+3$ throughout an interval if it exceeds $t+3$ at the endpoints. When $i=3,(*)$ requires $n \leq 2 t+11$, but we have assumed $n>4 t+10$. Since $n / 2-3>t+3$ when $n>2 t+12$, the inequality holds for $i=n / 2-1$ only if $n-t \leq\left\lceil\frac{3 i+7}{2}\right\rceil$, which requires $n \leq 4 t+10$. Thus $d_{i} \geq i+t+1$ for $3 \leq i \leq n / 2-1$ when $n>4 t+10$.

If $d_{2} \geq t+1$ and $d_{1} \geq t$, then Lemma 4.2 yields $t$ edge-disjoint perfect matchings in $G$. Hence we may assume $d_{2} \leq t$ or $d_{1} \leq t-1$; our remaining arguments are structural. Choose distinct $u, v \in V(G)$ so that $d_{G}(u)=d_{1}$ and $d_{G}(v)=d_{2}$.

If $d_{2} \geq t+1$, then $d_{1} \leq t-1$. Now any color at $u$ in $K_{n}$ cannot appear in $G-\{u\}$, since substituting the edge at $u$ for the edge of the same color in $G$ would yield a representing subgaph with larger minimum degree. Form $G^{\prime}$ from $G$ by adding $t-d_{1}$ of the edges incident to $u$ in $K_{n}$. Now $d_{G^{\prime}}(u)=t$, and $d_{G^{\prime}}(w) \geq d_{G}(w)$ for all $w$. By Lemma $4.2, G^{\prime}$ contains $t$ edge-disjoint perfect matchings. These are rainbow matchings in the edge-coloring, since each uses exactly one edge at $u$, and the colors on those edges appear nowhere else in $G^{\prime}$.

We may therefore assume $d_{2} \leq t$. Since $d_{3} \geq t+4$, any color appearing at $u$ or $v$ cannot occur in $G-\{u, v\}$, since substituting that edge for the edge of the same color in $G$ would yield a representing subgraph with lowest degrees lexicographically larger than $\left(d_{1}, d_{2}\right)$. It 
therefore suffices to find edges at $u$ and $v$ for the $t$ rainbow matchings and then complete those matchings using edges from $G$ at the remaining vertices. Let $E_{1}=\{u v\}$, and let $E_{2}, \ldots, E_{t}$ be disjoint rainbow pairs of nonincident edges at $u$ and $v$, if they exist. The $j$ th matching $M_{j}$ will contain $E_{j}$.

If $\left\{E_{1}, \ldots, E_{t}\right\}$ exist, then we use Lemma 4.2 to find $M_{j}$. For $1 \leq j \leq t$, let $G_{j}$ be the spanning subgraph whose edge set consists of $E_{j}$, all edges of $G-\{u, v\}$ not used in $M_{1}, \ldots, M_{j-1}$, and one more edge incident to $v$ and to the end other than $u$ of the edge incident to $u$ in $E_{j}$. Note that $d_{G_{j}}(u)=1$ and $d_{G_{j}}(v)=2$. Each vertex outside $\{u, v\}$ loses $j-1$ incident edges for the matchings and at most two more to $\{u, v\}$. With degrees $d_{1}^{\prime}, \ldots, d_{n}^{\prime}$ for $G_{j}$ and $d_{i} \geq i+t+1$ in $G$, we thus have $d_{1}^{\prime}=1, d_{2}^{\prime}=2$, and $d_{i}^{\prime} \geq i+t-j$ for $i \geq 3$. Since $j \leq t$, always $d_{i}^{\prime} \geq i$. Hence Lemma 4.2 yields a perfect matching $M_{j}$ in $G_{j}$. Since $d_{1}^{\prime}=1$ and $d_{2}^{\prime}=2$, the edges of $M_{j}$ incident to $\{u, v\}$ must be precisely the edges of $E_{j}$.

It remains only to find the pairs $E_{2}, \ldots, E_{t}$. For this we seek a matching in an auxiliary $X, Y$-bigraph $H$. Let $X=\left\{e_{1}, \ldots, e_{n-2}\right\}$ and $Y=\left\{f_{1}, \ldots, f_{n-2}\right\}$, where $\left\{e_{1}, \ldots, e_{n-2}\right\}$ and $\left\{f_{1}, \ldots, f_{n-2}\right\}$ are the sets of edges in $K_{n}-u v$ incident to $u$ and to $v$, respectively. Put $e_{i} f_{j} \in E(H)$ if and only if $e_{i}$ and $f_{j}$ have different colors and share no endpoint. We show that $H$ has a matching of size $t-1$; the edges of the matching are the desired pairs.

Let $c_{1}, \ldots, c_{2 n-4}$ be the vertex degrees in $H$, in nondecreasing order. By Theorem 4.1 with $k=n-t-1$, the proof is complete when $c_{n-t+1} \geq \frac{t-1}{2}$. Hence we may assume $c_{n-t+1}<\frac{t-1}{2}$. Say that a vertex in $H$ with degree at most $t / 2-1$ has low degree.

Let $e_{i}$ be a vertex of low degree in $H$, and let $a$ be the color on $e_{i}$. Excluding the edge incident to $e_{i}$ and at most $t / 2-1$ other edges leaves at least $n-2-t / 2$ edges at $v$ (other than $u v$ ) with color $a$. The same reasoning applies if $d_{H}\left(f_{j}\right) \leq t / 2-1$. Since $t<n / 4$, all low-degree vertices of $H$ in the same part have the same color.

If $X$ and $Y$ have both low-degree vertices, with different colors, then we now have at least $2 n-2-t$ edges incident to $\{u, v\}$ in these two colors. Since $n-t+1>2$, there are more than two low-degree vertices, and hence there are strictly more than $2 n-2-t$ edges in these two colors. Since only $2 n-3$ edges are incident to $\{u, v\}$ in $K_{n}$, this leaves at most $t-2$ edges, so at most $t$ colors are incident to $\{u, v\}$. At most $\left(\begin{array}{c}n-2 \\ 2\end{array}\right)$ colors appear on edges not incident to $\{u, v\}$, so there are at most $\left(\begin{array}{c}n-2 \\ 2\end{array}\right)+t$ colors, a contradiction.

Hence all the low-degree vertices in $H$ have the same color; call it $a$. Since at least $t+1$ colors must appear incident to $\{u, v\}$, at most $2 n-3-t$ vertices of $H$ can have color $a$, and at least $t-1$ have other colors. At least one part, say $X$ has at least $n-2-t / 2$ vertices of color $a$. Now $Y$ has at least $n-t+1$ such vertices (if all the low-degree vertices are in $Y$ ) or at least $n-2-t / 2$ such vertices (if both parts have low-degree vertices). In either case, select $t-1$ vertices of other colors; at most $t-1$ have been selected on each side. Since $n-2-t / 2>n-t+1 \geq t-1$ when $n \geq 2 t-2$, these $t-1$ vertices can be matched to vertices of color $a$ to obtain the desired matching in $H$. 


\section{Rainbow Spanning Trees}

We begin with the constructions for the lower bounds in Theorem 1.3. The general construction for the lower bound $\left(\begin{array}{c}n-2 \\ 2\end{array}\right)+t$ is just the special case of Proposition 2.4 when $q \geq n-1$ and hence $p=1$. We describe the special case here for clarity and to compare with the stronger constructions when $n \in\{2 t, 2 t+1\}$ (when $n<2 t$, the answer is $\left(\begin{array}{l}n \\ 2\end{array}\right)$ ).

Lemma 5.1. For positive integers $n$ and $t$ such that $t \leq 2 n-3$, there is an edge-coloring of $K_{n}$ using $\left(\begin{array}{c}n-2 \\ 2\end{array}\right)+t$ colors that does not have $t$ edge-disjoint rainbow spanning trees. When $n=2 t+1$, the construction improves to $\left(\begin{array}{c}n-2 \\ 2\end{array}\right)+2 t-1$ colors. When $n=2 t$, it improves to $\left(\begin{array}{c}n-2 \\ 2\end{array}\right)+3 t-3$, which equals $\left(\begin{array}{l}n \\ 2\end{array}\right)-t$ in that case.

Proof. We require $t \leq 2 n-3$ so that $K_{n}$ has at least $\left(\begin{array}{c}n-2 \\ 2\end{array}\right)+t$ edges. Fix two vertices $u$ and $v$. Use color 1 on all but $t-1$ edges joining $u$ or $v$ to the $n-2$ other vertices. Use distinct colors on the remaining edges. This coloring uses $\left(\begin{array}{c}n-2 \\ 2\end{array}\right)+t$ colors. Every rainbow spanning tree contains at least two edges incident to $\{u, v\}$. Since only $t-1$ edges incident to $u$ or $v$ have colors different from 1 and at least one of them must be used in each rainbow spanning tree, there are at most $t-1$ edge-disjoint rainbow spanning trees.

When $n=2 t+1$, any $t$ edge-disjoint spanning trees use all but $t$ edges. An edge-coloring with $2 t+1$ edges in one color therefore must have two edges of that color in one of the $t$ spanning trees, even if the other $\left(\begin{array}{l}n \\ 2\end{array}\right)-2 t-1$ edges have distinct colors. The number of colors in the construction is $\left(\begin{array}{l}n \\ 2\end{array}\right)-2 t$, which equals $\left(\begin{array}{c}n-2 \\ 2\end{array}\right)+2 t-1$ when $n=2 t+1$.

When $n=2 t$, let $t+1$ edges have color 1 and the other edges have distinct other colors; $\left(\begin{array}{l}n \\ 2\end{array}\right)-t$ colors are used. In this case, forming $t$ edge-disjoint spanning trees requires using all the edges, so the plentiful color contributes twice to one of the sets.

In the rest of this section, we prove the upper bounds on $r(n, t)$. In the main proof, we start with an edge-coloring of $K_{n}$ with $\left(\begin{array}{c}n-2 \\ 2\end{array}\right)+t+1$ colors. With this many colors, Lemma 5.4 guarantees that some representing subgraph of $K_{n}$ has a $2 t$-connected subgraph. In such a subgraph, a famous result of Nash-Williams (Theorem 5.2) guarantees $t$ edge-disjoint spanning trees.

Theorem 5.2. (Nash-Williams [21]) Every 2t-edge-connected graph contains $t$ edge-disjoint spanning trees.

We will extend these trees to edge-disjoint rainbow spanning trees in the full graph. It will be easy to extend to all but the last two vertices; absorbing the last two vertices requires applying Theorem 5.3 to an auxiliary bipartite graph.

Theorem 5.3. If $G$ is a bipartite graph with parts $X$ and $Y$, then $\alpha^{\prime}(G) \geq \min \{2 \delta(G),|X|,|Y|\}$. 
Theorem 5.3 follows from Theorem 4.1, but this folklore result also has a simple direct proof. If a maximum matching $M$ is smaller, then it omits vertices $x \in X$ and $y \in Y$. These vertices must have as neighbors both endpoints of some edge in $M$ (since the set of vertices omitted by $M$ is independent and $|M|<2 \delta(G)$ ), yielding an augmenting path of length 3 .

The $2 t$-edge-connected graph to which we will apply Theorem 5.2 will be obtained via the following lemma.

Lemma 5.4. Every graph $G$ with $n-\ell$ vertices, $\delta(G) \geq k$, and $|E(G)| \geq\left(\begin{array}{c}n-2 \\ 2\end{array}\right)+\frac{k}{2}+1-\ell(k-1)$ is $k$-edge-connected, where $n, k, \ell \in \mathbb{Z}$ satisfy $1 \leq k+1 \leq n-\ell$ and $n \geq k+6$.

Proof. Suppose that $G$ has a minimal edge-cut $F$ of size less than $k$. Since $\delta(G) \geq k$, each component of $G-F$ has size at least $k+1$, by a well-known elementary exercise. Hence $|E(G)| \leq\left(\begin{array}{c}k+1 \\ 2\end{array}\right)+\left(\begin{array}{c}n-\ell-k-1 \\ 2\end{array}\right)+k-1$. Since we are given $|E(G)| \geq\left(\begin{array}{c}n-2 \\ 2\end{array}\right)+\frac{k}{2}+1-\ell(k-1)$, it suffices to prove $f(\ell)<0$, where

$$
f(\ell)=\left(\begin{array}{c}
k+1 \\
2
\end{array}\right)+\left(\begin{array}{c}
n-\ell-k-1 \\
2
\end{array}\right)+k-1-\left(\begin{array}{c}
n-2 \\
2
\end{array}\right)-\frac{k}{2}-1+\ell(k-1) .
$$

The derivative of $f$ is $\frac{-1}{2}[2(n-\ell-k-1)-1]+k-1$, and $f^{\prime \prime}(\ell)=1$. Hence $f(\ell) \leq$ $\max \{f(0), f(n-k-1)\}$ when $0 \leq \ell \leq n-k-1$. Since $f(0), f(n-k-1)<0$, we also have $f(\ell)<0$, as desired.

The next lemma is the main case of the upper bound in Theorem 1.3. For a vertex $v$ in a graph $G$, let $\Gamma(v)$ denote the set of edges incident to $v$.

Lemma 5.5. If $n>2 t+\sqrt{6 t-\frac{23}{4}}+\frac{5}{2}$, then $r(n, t) \leq\left(\begin{array}{c}n-2 \\ 2\end{array}\right)+t$.

Proof. Note that $n \geq 2 t+3$ under this hypothesis when $t \geq 1$. It suffices to show that an edge-coloring of $K_{n}$ using exactly $\left(\begin{array}{c}n-2 \\ 2\end{array}\right)+t+1$ different colors has $t$ edge-disjoint rainbow spanning trees. Let $G$ be a representing subgraph.

If $G$ is disconnected, then $G$ has an isolated vertex $u$, since $\left(\begin{array}{c}n-2 \\ 2\end{array}\right)+1$ is the maximum number of edges in an $n$-vertex graph having at least two nontrivial components. Since $G-u$ is lacking only $n-1-t$ edges, in fact $G-u$ is 2-edge-connected. Choose $e \in \Gamma(u)-E(G)$. Let $e^{\prime}$ be the edge in $G$ having the same color as $e$. The graph $G-e^{\prime}+e$ is a connected representing subgraph. This proves the full claim when $t=1$ (completing the result of [4]). Henceforth we may assume $t \geq 2$, which yields $n \geq 2 t+6$.

Among all connected representing subgraphs of $K_{n}$, choose $G$ to lexicographically minimize $\left(m_{G}(1), \ldots, m_{G}(t)\right)$, where $m_{G}(t)$ is the number of vertices in $G$ with degree at most $t$. Call this $t$-tuple the $t$-vector of $G$, denoted $c_{t}(G)$; it will be relevant later in the proof.

Since $n>2 t+\sqrt{6 t-\frac{23}{4}}+\frac{5}{2}$ implies $\left(\begin{array}{c}2 t \\ 2\end{array}\right)+(n-2 t)(2 t-1)<\left(\begin{array}{c}n-2 \\ 2\end{array}\right)+t+1$, iteratively adding vertices joined to at most $2 t-1$ earlier vertices cannot produce enough edges to construct $G$. Hence $G$ has a subgraph $H$ with $\delta(H) \geq 2 t$ that is obtained from $G$ by iteratively deleting 
vertices with remaining degree at most $2 t-1$. If $\ell$ vertices are deleted to obtain $H$, then $|E(H)| \geq|E(G)|-\ell(2 t-1)=\left(\begin{array}{c}n-2 \\ 2\end{array}\right)+t+1-\ell(2 t-1)$. By Lemma 5.4 with $k=2 t$, the graph $H$ is $2 t$-edge-connected. Hence $|V(H)| \geq 2 t+1$ and $\ell \leq n-(2 t+1)$.

Index $V(G)-V(H)$ as $u_{1}, \ldots, u_{\ell}$ by choosing $u_{i}$ among the remaining vertices to have the most neighbors in $V(H) \cup\left\{u_{1}, \ldots, u_{i-1}\right\}$; let $d_{i}^{*}$ be that number of neighbors.

We claim that $d_{i}^{*} \geq t+1$ when $i \leq \ell-2$. Fix $i$, and let $b=d_{i}^{*}$. The choice of $u_{i}$ yields $d_{i+j}^{*} \leq b+j$ for $0 \leq j \leq \ell-i$. Let $k=\ell-i+1$. We have $|E(G)| \leq\left(\begin{array}{c}n-k \\ 2\end{array}\right)+\sum_{j=0}^{k-1}(b+j)=\left(\begin{array}{c}n-k \\ 2\end{array}\right)+k b+\left(\begin{array}{c}k \\ 2\end{array}\right)$. Since $\left(\begin{array}{c}n-2 \\ 2\end{array}\right)-\left(\begin{array}{c}n-k \\ 2\end{array}\right)=n(k-2)-\left(\begin{array}{c}k \\ 2\end{array}\right)-(k-3)$, the contradiction $\left(\begin{array}{c}n-k \\ 2\end{array}\right)+k b+\left(\begin{array}{c}k \\ 2\end{array}\right)<\left(\begin{array}{c}n-2 \\ 2\end{array}\right)+t+1=|E(G)|$ holds if and only if $n>k+2+\frac{k b-t}{k-2}$. Suppose $b \leq t$. If $k=3$, then $n>k+2+\frac{k b-t}{k-2}$, since $n \geq 2 t+6$ when $t \geq 2$. For $k \geq 4$, we have $k \leq \ell \leq n-(2 t+1)$, and hence $k+2+\frac{k b-t}{k-2} \leq n-2 t+1+t\left(\frac{k-1}{k-2}\right)<n$. Since $b \leq t$ yields a contradiction whenever $k \geq 3$, we obtain $d_{i}^{*} \geq t+1$ when $i \leq \ell-2$.

Let $G^{\prime}=G-\left\{u_{\ell-1}, u_{\ell}\right\}$. Since $H$ is $2 t$-edge-connected, $H$ has $t$ edge-disjoint spanning trees, by Theorem 5.2. Since $d_{i}^{*} \geq t+1$ when $i \leq \ell-2$, we can iteratively add $u_{1}, \ldots, u_{\ell-2}$ to $H$, letting $e_{1}^{i}, \ldots, e_{t}^{i}$ be distinct edges joining $u_{i}$ to $V(H) \cup\left\{u_{1}, \ldots, u_{i-1}\right\}$ in $G$. Adding $\left\{e_{j}^{1}, \ldots, e_{j}^{\ell-2}\right\}$ to the $j$ th spanning tree in $H$ (for each $j$ ) creates $t$ edge-disjoint spanning trees of $G^{\prime}$; call them $T_{1}, \ldots, T_{t}$. If also $d_{\ell-1}^{*}, d_{\ell}^{*} \geq t$, then we further augment $T_{1}, \ldots, T_{t}$, adding $t$ edges from $u_{\ell-1}$ and then from $u_{\ell}$ to obtain $t$ edge-disjoint rainbow spanning trees of $K_{n}$. Hence $d_{\ell-1}^{*}<t$ or $d_{\ell}^{*}<t$. By the choice of $u_{1}, \ldots, u_{\ell}$, we cannot have $d_{\ell-1}^{*}<t$ and $d_{\ell}^{*}>t$. This leaves two cases.

Case 1: $d_{\ell-1}^{*} \geq t+1$ and $d_{\ell}^{*} \leq t-1$. Add edges from $u_{\ell-1}$ to augment $T_{1}, \ldots, T_{t}$ into edge-disjoint spanning trees of $G-u_{\ell}$. Note that $\delta\left(G-u_{\ell}\right) \geq t+1$. Let $e$ be incident to $u_{\ell}$ in $K_{n}-E(G)$. Let $e^{\prime}$ be the edge in $G$ having the color of $e$, and let $F=G-e^{\prime}+e$. If $e^{\prime}$ is not incident to $u_{\ell}$, then its endpoints have degree at least $t+1$ in $G$. The first index where $c_{t}(F)$ and $c_{t}(G)$ differ is $d_{\ell}^{*}$, and its value in $c_{t}(F)$ is less than in $c_{t}(G)$ since $d_{F}\left(u_{\ell}\right)>d_{G}\left(u_{\ell}\right)$. This makes $c_{t}(F)$ lexicographically smaller than $c_{t}(G)$, contradicting the choice of $G$. Hence we may assume that the color of any edge incident to $u_{\ell}$ in $K_{n}-E(G)$ appears on no edge in $G-u_{\ell}$. For any edges $e_{1}, \ldots, e_{t}$ incident to $u_{\ell}$ in $K_{n}$, adding $e_{i}$ to the $i$ th spanning tree of $G-u_{\ell}$ completes $t$ edge-disjoint rainbow spanning trees of $K_{n}$, as desired.

Case 2: $d_{\ell-1}^{*} \leq t$ and $d_{\ell}^{*} \leq t$, with strict inequality for at least one. We have $h \leq 2 t-1$, where $h=d_{\ell-1}^{*}+d_{\ell}^{*}$. For $v \in V\left(G^{\prime}\right)$, we have $|E(G)| \leq\left(\begin{array}{c}n-3 \\ 2\end{array}\right)+d_{G^{\prime}}(v)+h$. Since $|E(G)| \geq\left(\begin{array}{c}n-2 \\ 2\end{array}\right)+t+1$ and $n \geq 2 t+6$, we obtain $d_{G}(v) \geq d_{G^{\prime}}(v) \geq 3 t+4-h$. Since $h \leq 2 t-1$, we have $d_{G}(v) \geq t+5$ for $v \notin\left\{u_{\ell-1}, u_{\ell}\right\}$. If some edge $e$ incident to $u_{\ell-1}$ or $u_{\ell}$ in $K_{n}-E(G)$ has the same color as an edge $e^{\prime}$ in $G^{\prime}$, then let $F=G-e^{\prime}+e$, as in Case 1 . Now $d_{F}(v)>d_{G}(v)$ when $v$ is at least one of $\left\{u_{\ell-1}, u_{\ell}\right\}$, while $d_{F}(v) \geq t+4$ for all other vertices. Again $c_{t}(F)$ is lexicographically smaller than $c_{t}(G)$, contradicting the choice of $G$. Hence we may assume that the color of any edge incident to $\left\{u_{\ell-1}, u_{\ell}\right\}$ in $K_{n}-E(G)$ appears on no edge in $G^{\prime}$. Our task now is to pick pairs of edges to extend $T_{1}, \ldots, T_{t}$ to reach $u_{\ell-1}$ and $u_{\ell}$.

Let $e_{1}, \ldots, e_{n-2}$ be the edges incident to $u_{\ell-1}$ in $K_{n}-\left\{u_{\ell}\right\}$, and let $e_{1}^{\prime}, \ldots, e_{n-1}^{\prime}$ be the edges 
incident to $u_{\ell}$ in $K_{n}$. Construct an auxiliary bipartite graph $H$ with partite sets $\left\{e_{1}, \ldots, e_{n-2}\right\}$ and $\left\{e_{1}^{\prime}, \ldots, e_{n-1}^{\prime}\right\}$ such that $e_{i} e_{j}^{\prime} \in E(G)$ if and only if $e_{i}$ and $e_{j}^{\prime}$ have different colors in $K_{n}$. If $\alpha^{\prime}(H) \geq t$, then a matching of size $t$ in $H$ corresponds to $t$ pairs of edges incident to $\left\{u_{\ell}, u_{\ell-1}\right\}$ such that the edges in each pair have different colors. Since these colors do not appear on edges of $G^{\prime}$, adding one of these pairs to each of $T_{1}, \ldots, T_{t}$ yields the desired $t$ edge-disjoint rainbow spanning trees of $K_{n}$.

Since $n \geq 2 t+6$, the partite sets of $H$ have size at least $2 t+4$. If also $\delta(H) \geq \frac{t}{2}$, then Theorem 5.3 yields $\alpha^{\prime}(H) \geq t$. Hence we may assume $\delta(H) \leq \frac{t-1}{2}$. Give each vertex of $H$ its color in the edge-coloring of $K_{n}$. Let $z$ be a vertex of minimum degree in $H$, and let $a$ be its color. Let $X$ and $X^{\prime}$ be the partite sets of $H$, named so that $z \in X$. Let $k$ and $k^{\prime}$ count the vertices with color $a$ in $X$ and $X^{\prime}$, respectively. Since $d_{H}(z)=\left|X^{\prime}\right|-k^{\prime}$ and $n \geq 2 t+6$, we have $k^{\prime} \geq n-2-\frac{t-1}{2} \geq t$.

If $|X|-k \geq t$, then $\alpha^{\prime}(H) \geq t$ by matching $t$ vertices of color $a$ in $X^{\prime}$ into $X$. Hence $k>|X|-t \geq t$. Now each part has at least $t$ vertices with color $a$. Since $|E(G)| \geq\left(\begin{array}{c}n-2 \\ 2\end{array}\right)+t+1$, there are also edges of at least $t$ colors other than $a$ incident to $\left\{u_{\ell-1}, u_{\ell}\right\}$. Now $t$ such vertices of $H$ can be matched to vertices with color $a$ to obtain $\alpha^{\prime}(H) \geq t$, no matter how those vertices are distributed to $X$ and $X^{\prime}$.

For a set $S$ of vertices in a graph $H$, let $N_{H}(S)$ denote the set of vertices with neighbors in $S$. In proving the upper bound for the case $n=2 t$, we use Hall's Theorem [15] stating that an $X, Y$-bigraph $H$ has a matching covering $X$ if and only if $\left|N_{H}(S)\right| \geq|S|$ for all $S \subseteq X$.

Lemma 5.6. If $n=2 t$, then $r(n, t) \leq\left(\begin{array}{l}n \\ 2\end{array}\right)-t$.

Proof. We use induction on $t$. For $t=1$ the claim is trivial. For $t=2$, any edge-coloring of $K_{4}$ with five colors decomposes into two rainbow spanning paths. For $t>2$, we may assume that the edge-coloring uses exactly $\left(\begin{array}{c}2 t \\ 2\end{array}\right)-t+1$ colors. A color appearing on only one edge (or the edge itself) is singleton. Let $d^{\prime}(v)$ be the number of singletons incident to $v$. If $k$ colors are non-singleton, then at most $\left(\begin{array}{c}2 t \\ 2\end{array}\right)-2 k$ edges are singleton. Hence at most $\left(\begin{array}{c}2 t \\ 2\end{array}\right)-k$ colors are used, so $k \leq t-1$, and at least $\left(\begin{array}{c}2 t \\ 2\end{array}\right)-2(t-1)$ colors are singleton. Thus $\sum_{v \in V\left(K_{2 t}\right)} d^{\prime}(v) \geq 2\left[\left(\begin{array}{c}2 t \\ 2\end{array}\right)-2(t-1)\right]=2 t(2 t-3)+4$. We consider two cases.

Case 1: For some vertex $u$, all incident edges are singleton. Some two edges have the same color, so $d^{\prime}(v) \leq 2 t-2$ for some vertex $v$. If every such vertex has at most $t-1$ incident singletons, then $d^{\prime}(v) \leq t-1$ and also $d^{\prime}(x) \leq t-1$ when $x v$ is non-singleton. With $d^{\prime}(x) \leq 2 t-1$ when $x v$ is singleton, $\sum_{w} d^{\prime}(w) \leq(t+1)(t-1)+(t-1)(2 t-1)=(t-1) 3 t$, which contradicts $\sum_{v} d^{\prime}(v) \geq 2 t(2 t-3)+4$. Hence $t \leq d^{\prime}(w) \leq 2 t-2$ for some vertex $w$.

Let $R=V\left(K_{2 t}\right)-\{u, w\}$. Since $d^{\prime}(u)=2 t-1$ implies that $u w$ is singleton, deleting $u$ and $w$ eliminates at most $4 t-4$ colors. Hence at least $\left(\begin{array}{c}2 t-2 \\ 2\end{array}\right)-(t-1)+1$ colors are used within $R$. Since $|R|=2 t-2$, by the induction hypothesis the edge-coloring induced by $R$ decomposes into rainbow spanning trees $T_{1}, \ldots, T_{t-1}$. Let $\mathbf{T}=\left\{T_{1}, \ldots, T_{t-1}\right\}$. 
Let $e_{1}, \ldots, e_{p}$ be the non-singleton edges incident to $w$; these join $w$ to $R$. Since $d^{\prime}(w) \geq t$, we have $p \leq t-1$. We will match $p-1$ of these edges to trees in $\mathbf{T}$ so that the color on each matched edge does not appear in the tree it is matched to. This extends these trees to $w$. Among the remaining edges incident to $u$ and $w$ we will find a spanning tree $T_{t}$ of $K_{2 t}$, edges to extend the unmatched trees to $w$, and edges to extend each tree in $\mathbf{T}$ to $u$.

To find the matching, consider an auxiliary bipartite graph $H$ with parts $\left\{e_{1}, \ldots, e_{p}\right\}$ and T. Make $e_{i}$ and $T_{j}$ adjacent in $H$ if and only if the color on $e_{i}$ appears on no edge in $T_{j}$. To prove $\alpha^{\prime}(H) \geq p-1$, we use Hall's Theorem [15].

If $\alpha^{\prime}(H)<p$, then let $S$ be a minimal subset of $\left\{e_{1}, \ldots, e_{p}\right\}$ such that $\left|N_{H}(S)\right|<|S|$; hence $\left|N_{H}(S)\right|=|S|-1$. Let $C$ be the set of colors on edges in $S$. Let $l=|S|$ and $m=|C|$, so $m \leq l$. All colors in $C$ appear in all $t-1-(l-1)$ trees outside $N_{H}(S)$. Hence these colors cover at least $m(t-l)+l$ edges in $K_{2 t}$. Deleting all but one edge of each color in $C$ keeps at least one edge of each color, so $\left(\begin{array}{c}2 t \\ 2\end{array}\right)-m(t-l)-l+m \geq\left(\begin{array}{c}2 t \\ 2\end{array}\right)-t+1$. This inequality simplifies to $(m-l) l \geq(m-1)(t-1)$. If $m \geq 2$, then $m-1 \geq m-l$ yields $l \geq t-1 \geq p$, which implies $\alpha^{\prime}(H) \geq p-1$. If $m=1$, then the one color in $C$ appears in all $t-l$ trees outside $N_{H}(S)$ and on $S$, so it appears on $t$ edges. With $\left(\begin{array}{c}2 t \\ 2\end{array}\right)-t+1$ colors, all other edges are singleton. Hence $l=p$, and again $\alpha^{\prime}(H) \geq p-1$.

Extend $p-1$ trees in $\mathbf{T}$ to $w$ using this matching. The color of the unmatched edge $e_{j}$ is on no other unassigned edge. Since $p \leq t-1$ and $|R|=2 t-2$, we can pick $t-1$ unassigned edges from $w$ to $R$ including $e_{j}$, add $u w$, and add edges from $u$ to the $t-1$ other vertices of $R$ to form a rainbow spanning tree $T_{t}$. Assign the other $t-p$ edges at $w$ to the trees in $\mathbf{T}$ not yet extended, and match the remaining $t-1$ edges at $u$ to these trees arbitrarily.

Case 2: Every vertex is incident to a non-singleton edge. With $d^{\prime}(v) \leq 2 t-2$ for all $v$, at least four vertices have only one non-singleton incident edge, since $\sum_{v} d^{\prime}(v) \geq 2 t(2 t-3)+4$. Let $u$ and $w$ be two such vertices. Deleting $u$ and $w$ eliminates at most $4 t-4$ colors, so the remaining edge-coloring $H$ has at least $\left(\begin{array}{c}2 t-2 \\ 2\end{array}\right)-(t-1)+1$ colors. Again by the induction hypothesis, $H$ decomposes into edge-disjoint rainbow spanning trees $T_{1}, \ldots, T_{t-1}$.

Let $e$ and $e^{\prime}$ be the non-singleton edges incident to $u$ and $w$, respectively, and let $a$ be the color on $e$. If $a$ appears $t$ times in the full coloring, then the remaining edges are singleton. Hence if $e \neq e^{\prime}$, then $a$ appears at most $t-2$ times in $H$ (whether or not $e$ and $e^{\prime}$ have the same color), so some tree among $T_{1}, \ldots, T_{t-1}$ does not use $a$. Reserve $e$ to extend that tree to $u$. Now complete the construction as in Case 1 with $p=1$ and $e_{1}=e^{\prime}$.

If $e=e^{\prime}$, then $e=u w$ and all edges incident to $u$ and $w$ have distinct colors. Since $a$ appears elsewhere only in $T_{1}, \ldots, T_{t-1}$, again the construction of Case 1 works, since $e$ goes into the added tree $T_{t}$.

Lemmas 5.1, 5.5, and 5.6 comprise the proof of Theorem 1.3. 


\section{References}

[1] S. Akbari and A. Alipour, Multicolored trees in complete graphs. J. Graph Theory 54 (2007), 221-232.

[2] S. Akbari, O. Etesami, H. Mahini, and M. Mahmoody, On rainbow cycles in edge colored complete graphs. Australas. J. Combin. 37 (2007), 33-42.

[3] C. Berge, Graphs and Hypergraphs. (North-Holland, 1973).

[4] A. Bialostocki and W. Voxman, On the anti-Ramsey numbers for spanning trees. Bull. Inst. Combin. Appl. 32 (2001), 23-26.

[5] R.A. Brualdi and S. Hollingsworth, Multicolored trees in complete graphs. J. Combin. Theory Ser. B 68 (1996), 310-313.

[6] J.M. Carraher, S.G. Hartke, and P. Horn, Edge-disjoint rainbow spanning trees in complete graphs. Submitted, 2013.

[7] H. Chen, X. Li, and J. Tu, Complete solution for the rainbow numbers of matchings. Discrete Math. 309 (2009), 3370-3380.

[8] V. Chvátal, On Hamilton's ideals. J. Combinatorial Theory Ser. B 12 (1972), 163-168.

[9] P. Erdős, M. Simonovits, and V. Sós, Anti-Ramsey theorems, in Infinite and Finite Sets (Colloq. Keszthely 1973). Colloq. Math. Soc. Janos Bolyai 10 (1975), 633-643.

[10] S. Fujita, C. Magnant, and K. Ozeki, Rainbow generalizations of Ramsey theory: a survey. Graphs Combin. 26 (2010), 1-30.

[11] S. Fujita, A. Kaneko, I. Schiermeyer, and K. Suzuki, A rainbow k-matching in the complete graph with r colors. Electr. J. Combinatorics 16 (2009), Paper \#R51.

[12] A. Gyárfás, M. Ruszinkó, G.N. Sárközy, and R.H. Schelp, Long rainbow cycles in proper edge-colorings of complete graphs. Australas. J. Combin. 50 (2011), 45-53.

[13] R. Haas and M. Young, The anti-Ramsey number of perfect matching. Discrete Math. 312 (2012), 933-937.

[14] P. Hall, On representatives of subsets. J. London Math. Soc. 10 (1935), 26-30.

[15] P. Horn, Rainbow spanning trees in complete graphs colored by matchings. Preprint, 2013. 
[16] S. Jahanbekam and D.B. West, New lower bounds on matching numbers of general and bipartite graphs, Congressus Numer. 218 (2013), 57-59.

[17] T. Jiang and D. B. West, Edge-colorings of complete graphs that avoid polychromatic trees, Discrete Math. 274 (2004), 137-145.

[18] A. Kaneko, M. Kano, and K. Suzuki, Three edge disjoint multicolored spanning trees in complete graphs. Preprint, 2003.

[19] T.D. LeSaulnier and D.B. West, Rainbow edge-coloring and rainbow domination. Discrete Math. 313 (2013), 2020-2025.

[20] J.J. Montellano-Ballesteros and V. Neumann-Lara, An anti-Ramsey theorem on cycles. Graphs Combin. 21 (2005), 343-354.

[21] C. St. J. A. Nash-Williams, Edge disjoint spanning trees of finite graphs. J. London Math. Soc. 36 (1961), 445-450.

[22] I. Schiermeyer, Rainbow numbers for matchings and complete graphs, Discrete Math. 286 (2004), 157-162. 